\title{
Distances of Galactic supernova remnants
}

\author{
Hui Zhu and Wenwu Tian \\ National Astronomical Observatories, CAS, Beijing, China
}

\begin{abstract}
Supernova remnants (SNRs) play a key role in understanding supernovae explosion mechanisms, exploring the likely sources of Galactic cosmic rays and the chemical enrichment of interstellar medium (ISM). Reliable distance determinations to Galactic SNRs are key to obtain their basic parameters, such as size, age, explosion energy, which helps us to study their environment and interstellar medium. We review the methods to determine the distances to SNRs and highlight the kinematic distance measurement by Hi absorption and CO emission observations.
\end{abstract}

Keywords. ISM: kinematics and dynamics, ISM: supernova remnants

\section{The empirical surface brightness $(\Sigma)$ - diameter(D) relation}

The theoretical and observational studies indicate that there should be a relationship between surface brightness and diameter of an SNR, $\Sigma=a D^{\beta}$. However, the validity of this relation has not yet been universally accepted. The error in using this method can reach about $40 \%$ of the measured distance (Case \& Bhattacharya 1998). The reasons to explain dispersions in $\Sigma-D$ relation (Guseinov et al. 2003) include: 1) The wide range of explosion energies of supernovae. 2) The influence of ISM around SNRs. Normally, denser ISM will lead to higher surface brightness. 3) The influence of neutron star within an SNR. 4) The distance and diameters uncertainty in calibrators.

A frequently used relation was derived by analyzing 36 Galactic shell SNRs (Case \& Bhattacharya 1998). The derived slope was $=-2.38$. Guseinov et al. (2003) showed a two slopes $\Sigma-D$ relation with a turning point at $\mathrm{D}=36.5$ pc. For $\mathrm{D}>36.5 \mathrm{pc}, \beta=$ -5.99. For $\mathrm{D}<36.5 \mathrm{pc}, \beta=-2.47$. Pavlović et al. (2013) used orthogonal regression to replace the previous used vertical regression and the newly calculated slope, $\beta=-4.8$, is significantly steeper than those in previous works.

\section{The kinematic distance}

Kinematic distance measurement is based on an assumption that rotation curve of the Milk Way is flat outside the bulge region. For objects that follow the rotation curve well, we can derive their distances from the observed line-of-sight velocity (e.g. Hi clouds). $21 \mathrm{~cm}$ line observation toward SNRs is used to build Hi absorption spectra. Cold gases between observer and SNRs will lead to absorption, gases behind an SNR will not be seen in this SNR's absorption spectrum (see Figure 1). Therefore, the Hi absorption features are used to give a lower distance restriction and the emissions without absorptions are used to derive an upper distance restriction. For SNRs showing continuum emission and absorption features, their positions are given by the velocity at which the continuous absorption stops. Tian et al. (2007) presented an improved method (combining HI and CO lines's analysis) to build $21 \mathrm{~cm}$ HI absorption spectrum against a background extended radio source. In comparison with the traditional method-selecting background beside the source, they let the background surround the source directly which can minimize 


\section{Case 1: See HI emission but not absorption in $A$ against $B$ \\ Case 2: see $\mathrm{HI}$ emission and absorption in $\mathrm{A}$ against $B$}

\section{Case 3: see $\mathrm{HI}$ emissions from both $\mathrm{A}$ and $\mathrm{C}$, but only see absorption in A against $\mathrm{B}$}

\section{Continuum (Radio) Source:}

\section{Eyes}

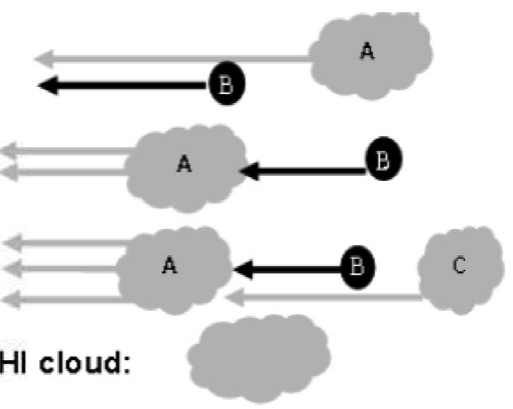

Figure 1. Three cases of relative positions between continuum source and Hi clouds.

the possibility of false absorption spectrum. In addition, they use CO spectrum toward target source and the Hi absorption spectra of other nearby bright continuum sources to understand absorption spectrum of an SNR better. Using this method, they showed new or revised distances for SNRs, e.g., G18.8+0.3, Kes 69, 73 \& 75, W41, CTB 37 \& 109, Tycho's SNR (Tian \& Leahy 2008a, 2008b, 2011, 2013; Leahy \& Tian 2008).

\section{Other methods}

Kassim et al. (1994) found that X-ray observations could be used to calculated the Sedov distance of shell type SNRs which are in the adiabatic expansion phase. Based on the X-ray data from ROSAT observations, they derived Sedov distances to 10 SNRs successfully. Some SNRs are associated with distance-known objects, such as pulsar, HiI region, CO cloud (e.g. Vela, Green 1984). Errors in this method are caused by not only the distance's errors of the associated objects, but also the uncertainty of such association. For nearby SNRs (e.g. Crab nebula), proper motions and radial velocity can be used to derive an expansion distance (Green 1984). Extinction measurement based on red clump stars has been used to measure distance (Güver et al. 2010). For infrared or optical bright SNRs, extinction $A_{V}$ can be measured by intensity ratio of $H_{\alpha}$ and $H_{\beta}$, the SII multiplet ratios at $\sim 1032.0 \mathrm{~nm}$ and $\sim 406.8 \mathrm{~nm}$, IR transitions of Fe[II] at 1.6435 and $1.2567 \mu \mathrm{m}$ etc. (Güver \& Özel 2009). For X-ray bright SNRs, we can derive the column density of hydrogen by X-ray absorption. Then hydrogen column density $\left(\mathrm{N}_{\mathrm{H}}\right)$ can be converted to extinction by the $\mathrm{N}_{\mathrm{H}}-\mathrm{A}_{\mathrm{V}}$ relation.

\section{References}

Case, G. L. \& Bhattacharya, D. 1998, ApJ, 30, 490

Green, D. A. 1984, MNRAS, 209, 449

Guseinov, O. H., Ankay, A., Sezer, A., \& Tagieva, S. O. 2003, A\&AT, 22, 273

Güver, T. \& Özel, F. 2009, MNRAS, 400, 2050

Güver, T., Özel, F., Cabrera-Lavers, A., \& Wroblewski, P., 2009, ApJ, 712, 964

Kassim, N. E., Hertz, P., van Dyk, S. D., \& Weiler, K. W. 1994, ApJ, 427, 95

Leahy, D. A. \& Tian, W. W. 2008, AJ, 135, 167

Pavlović, M. Z., Urošević, D., Vukotić, B., Arbutina, B., \& Göker, Ü. D. 2013, ApJS, 204, 4

Tian, W. W., Leahy, D. A., \& Wang, Q. D. 2007, A\& A, 474, 541

Tian, W. W. \& Leahy, D. A. 2008a, ApJ, 679, 85

Tian, W. W. \& Leahy, D. A. 2008b, MNRAS, 391, 54

Tian, W. W. \& Leahy, D. A. 2011, ApJ, 729, 15

Tian, W. W. \& Leahy, D. A. 2013, MNRAS, 421, 2593 